\title{
ON NONEXPANSIVE MAPPINGS ${ }^{1}$
}

\author{
L. A. KARLOVITZ
}

\begin{abstract}
A generalized Hilbert space property is used to analyze nonexpansive mappings in certain settings. In particular it is shown that in $l_{1}$ and in the important, recently defined, space $J_{0}$, a nonexpansive self-mapping of a bounded weak* closed convex subset has a fixed point.
\end{abstract}

1. We are concerned mainly with the existence of fixed points for nonexpansive mappings. More precisely, let $X$ be a (possibly nonreflexive) Banach space. Let $C$ be a bounded closed convex subset of $X$. If $X$ is nonreflexive, we shall assume that it is the conjugate of a separable space and that $C$ is weak* closed. Let $T: C \rightarrow C$ satisfy $\|T x-T y\| \leqslant\|x-y\|$ for all $x, y \in C$. Does there exist an $x_{0} \in C$ so that $T x_{0}=x_{0}$ ? To a lesser extent, we are also concerned with the iterative construction of fixed points.

Such problems have received considerable attention during the past decade. (Basic existence theorems for classes of reflexive spaces: Browder [2], Göhde [4], Kirk [7]. Iterative constructions related to the present note: Browder and Petryshyn [3], Opial [9].) They have been studied basically in reflexive spaces. Many of the results rely on the use of geometric properties of the space which are patterned after the geometry of Hilbert space.

This is also our present approach. We propose to extend the theory by making use of a geometric notion, which is a generalization of a Hilbert space property. In particular, this permits us to extend the theory to certain nonreflexive spaces.

2. In a general Banach space we say (Birkhoff [1], James [5]) that $w$ is orthogonal to $v, w \perp v$, if $\|w\| \leqslant\|w+\lambda v\|$ for all scalars $\lambda$. In general, $\perp$ is not symmetric. (Indeed, symmetry characterizes Hilbert spaces for dimension strictly greater than 2.) We shall say that the relation $\perp$ is approximately symmetric if for each $x \in X$ and $\varepsilon>0$ there exists a closed linear subspace $U=U(x, \varepsilon)$ so that

(1) $U$ has finite codimension, and

(2) $\|u\| \leqslant\|u+\lambda x\|$ for each $u \in U,\|u\|=1$, and each $\lambda,|\lambda| \geqslant \varepsilon$.

In case $\perp$ is symmetric, we can choose $U=\{u: f(u)=0\}$ where $f$ is a linear functional such that $\|f\|=1$ and $f(x)=\|x\|$. Then $x \perp u$ for each $u \in U$; and, by the symmetry, $u \perp x$ for each $u \in U$, which is stronger than (2). Furthermore, the linear projection $P$, of $X$ onto $U$, defined by $P(u+\lambda x)$ $=u$ has norm 1 . Analogously, if (2) is satisfied, the same projection, now of

Presented to the Society, October 29, 1974; received by the editors October 24, 1974.

AMS (MOS) subject classifications (1970). Primary 47H10; Secondary 46B05.

${ }^{1}$ This research was carried on, in part, while the author was visiting the University of Brasilia. 
the linear span of $x$ and $U$ onto $U$, has norm less than or equal to $1 / 1-\varepsilon$.

If $X$ is a conjugate space we shall say that the relation $\perp$ is weak* approximately symmetric if, in addition to (1) and (2), $U$ can be chosen to be weak* closed. We shall say that the relation is uniformly approximately symmetric (uniformly weak* approximately symmetric) if it is approximately symmetric (weak* approximately symmetric) and (2) is replaced by the stronger condition

$$
\begin{aligned}
& \|u\| \leqslant\|u+\lambda x\|-\delta, \text { for some } \delta=\delta(x, \varepsilon)>0 \text { for each } \\
& u \in U,\|u\|=1, \text { and each } \lambda,|\lambda| \geqslant \varepsilon .
\end{aligned}
$$

In terms of the projection defined above, $\left(2^{\prime}\right)$ requires that it actually decrease the norms of certain elements by a uniform amount.

It can be readily shown that if $X$ has a uniformly convex unit ball, then approximate symmetry and uniform approximate symmetry are equivalent.

EXAMPLE 1. If $X$ is a Hilbert space then $\perp$ is symmetric and the unit ball is uniformly convex; hence, $\perp$ is uniformly approximately symmetric.

EXAMPLE 2 . In $l_{1}$ the relation $\perp$ is uniformly weak* approximately symmetric. To see this, given $x=\left(\xi_{i}\right)$, choose $N$ so large that $\Sigma_{1}^{N}\left|\xi_{i}\right| \geqslant 3\|x\| / 4$. For each $\varepsilon>0$ let $U(x, \varepsilon)=\left\{u=\left(\eta_{i}\right): \eta_{i}=0, i=1, \ldots, N\right\}$. Then we readily observe that for each $u \in U,\|u+\lambda x\|-\|u\| \geqslant|\lambda|\|x\| / 2$, which implies the assertion.

FURTHER EXAMPLES. In the important, newly defined, space $J_{0}$ (James [6], Lindenstrauss and Stegall [8]) the relation $\perp$ is also uniformly weak* approximately symmetric. In the classical spaces $l_{p}, 1<p<\infty, \perp$ is uniformly approximately symmetric. The argument in both cases is a modification of the one for $l_{1}$ above. On the other hand, in both $c_{0}$ and $L_{p}, p \neq 2, \perp$ fails to be even approximately symmetric. We state the former without proof, and the latter is discussed in the Remark following Theorem 2 below.

Our main result which relates these geometric notions to fixed points for nonexpansive mappings is

THEOREM 1. Let $X$ be a reflexive Banach space (resp., the conjugate of $a$ separable Banach space). Let $C$ be a bounded convex closed (resp., weak* closed) subset of $X$. Let the mapping $T: C \rightarrow C$ satisfy $\|T x-T y\| \leqslant\|x-y\|$ for all $x, y \in C$. Suppose that the relation $\perp$ is uniformly approximately symmetric (resp., uniformly weak* approximately symmetric) in $X$. Then there exists $x_{0} \in C$ so that $T x_{0}=x_{0}$.

Furthermore, if $T$ is asymptotically regular (i.e., if for each $x \in C$, $\left\|T^{n+1} x-T^{n} x\right\| \rightarrow 0$ as $\left.n \rightarrow \infty\right)$ then for each $x \in C$ the sequence $\left\{T^{n} x\right\}$ converges weakly (resp., weak*) to some $x_{0} \in C$ and $T x_{0}=x_{0}$.

The conclusions of the theorem are known for the case that $X$ is a Hilbert space, and more generally for $l_{p}, 1<p<\infty$ (Opial [9]). However, other of its applications, including the following, seem to be new

Corollary. Let $C$ be a weak* closed convex bounded subset of $l_{1}$ or of $J_{0}$. Let $T: C \rightarrow C$ be a nonexpansive mapping. Then $T$ has a fixed point in $C$.

As a by-product of these investigations, we have

THEOREM 2. Let $X$ be a separable reflexive Banach space (resp., the conjugate 
of such a space). Then for each weakly (resp., weak*) convergent sequence $\left\{x_{n}\right\}$ with limit $x_{\infty}$ we have

$$
\lim \inf \left\|x_{n}-x_{\infty}\right\|<\lim \inf \left\|x_{n}-x\right\|,
$$

for each $x \neq x_{\infty}$, if and only if the relation $\perp$ is uniformly approximately symmetric (resp., uniformly weak* approximately symmetric).

Moreover, if the relation $\perp$ is approximately symmetric (resp., weak* approximately symmetric) then

$$
\lim \inf \left\|x_{n}-x_{\infty}\right\| \leqslant \lim \inf \left\|x_{n}-x\right\|,
$$

for each $x \in X$, but not conversely in general.

REMARK. The importance of inequality (3) in the investigation of fixed points of nonexpansive mappings is noted in Opial [9]. However, his method involves the abstraction of Hilbert space properties to a class of spaces which are substantially different from the present ones. His method demands: (i) reflexivity, (ii) a weakly continuous duality mapping, and (iii) a uniformly convex unit ball. He also shows that $\left(3^{\prime}\right)$ does not hold for all reflexive spaces with a uniformly convex unit ball by exhibiting a sequence in $L_{p}, 1<p<\infty$, $p \neq 2$, which fails to satisfy $\left(3^{\prime}\right)$. From this example and Theorem 2 it follows that in these spaces $L_{p}$ the relation $\perp$ is not approximately symmetric.

3. Proof of Theorem 1, part 1. By a standard argument, there exists a sequence $\left\{x_{n}\right\}$ in $C$ so that $\left\|T x_{n}-x_{n}\right\| \rightarrow 0$. After possible extraction of a subsequence, we may assume that the sequence converges weakly (weak*) to some $z \in C$ and $\lim \left\|x_{n}-z\right\|=r$. We let $w=T z-z$. If either $r=0$ or $w=0$ then $T z=z$, and we are finished. Hence we assume that $r>0$ and $w \neq 0$. We let $\varepsilon=1 / 2 r$. By hypothesis, there exists a closed (weak* closed) linear subspace $U$ so that (1) and (2') are satisfied. This is equivalent to

$$
\begin{aligned}
& |\lambda| \leqslant\|w+\lambda u\|-|\lambda| \delta, \text { for some } \delta>0 \text { and for each } u \in U, \\
& \|u\|=1, \text { and each } \lambda,|\lambda| \leqslant 2 r .
\end{aligned}
$$

Furthermore, the subspace spanned by $w$ and $U$ has a finite dimensional complement $V$. Thus for each $n$,

$$
x_{n}-z=\lambda_{n} w+u_{n}+v_{n}, \quad u_{n} \in U, \quad v_{n} \in V .
$$

Using the finite dimensionality of $V$ and the convergence of $x_{n}-z$ we observe readily that $\lambda_{n},\left\|v_{n}\right\| \rightarrow 0$, and hence $\left\|u_{n}\right\| \rightarrow r$. Therefore $\left\|u_{n}\right\| / 1+\lambda_{n}$ $\leqslant 2 r$ for sufficiently large $n$, and by (4) and (5),

$$
\begin{aligned}
\left\|x_{n}-T z\right\| & \geqslant\left\|x_{n}-z+z-T z\right\|=\left\|\left(1+\lambda_{n}\right) w+u_{n}+v_{n}\right\| \\
& \geqslant\left|1+\lambda_{n}\right|\left\|w+\left(\left\|u_{n}\right\| / 1+\lambda_{n}\right) u_{n} /\right\| u_{n}\|\|-\left\|v_{n}\right\| \\
& \geqslant\left\|u_{n}\right\|(1+\delta)-\left\|v_{n}\right\| .
\end{aligned}
$$

On the other hand, by the nonexpansiveness of $T$,

$$
\left\|x_{n}-T z\right\| \leqslant\left\|T x_{n}-T z\right\|+\left\|x_{n}-T x_{n}\right\| \leqslant\left\|x_{n}-z\right\|+\left\|x_{n}-T x_{n}\right\| .
$$

Combining the last two inequalities, and using $\left\|x_{n}-T x_{n}\right\|,\left\|v_{n}\right\| \rightarrow 0$ and $\left\|u_{n}\right\|$, $\left\|x_{n}-z\right\| \rightarrow r$, we arrive at the contradiction: $r \geqslant r(1+\delta)$. Thus $r>0$ and $w \neq 0$ is impossible. This finishes the proof of the first part of Theorem 1 .

Proof of Theorem 2. Suppose that $\perp$ is uniformly (weak*) approximately 
symmetric. Suppose $\left\{x_{n}\right\}$ converges weakly (weak*) to $x_{\infty}$. Let $\lim \inf \left\|x_{n}-x_{\infty}\right\|$ $=s$. If $s=0$, (3) follows. If $s>0$, for $x \neq x_{\infty}$, we extract a subsequence $\left\{x_{n^{\prime}}\right\}$, so that $\lim \left\|x_{n^{\prime}}-x_{\infty}\right\|$ exists and $\lim \left\|x_{n^{\prime}}-x\right\|=\lim \inf \left\|x_{n^{\prime}}-x\right\|$. Then with very minor modifications in the proof of Theorem 1, part 1, we can again derive inequality (6), with $T z$ replaced by $x, z$ replaced by $x_{\infty}$, and $x_{n}$ replaced by $x_{n}{ }^{\prime}$. From this we conclude

$$
\lim \left\|x_{n^{\prime}}-x\right\| \geqslant \lim \left\|x_{n^{\prime}}-x_{\infty}\right\|(1+\delta),
$$

which implies (3).

Now suppose that $\perp$ is not uniformly (weak*) approximately symmetric. Choose $x$ and $\varepsilon>0$ so that (1) and (2') fail to be satisfied. Choose a sequence of linear functionals $\left\{f_{n}\right\}$ which are dense in $X^{*}$ (in the space of which $X$ is the conjugate). Let $U_{n}=\left\{x: f_{j}(x)=0, j=1, \ldots, n\right\}$. Since (2') is not satisfied, we can choose $u_{n} \in U_{n},\left\|u_{n}\right\|=1$, and $\lambda_{n},\left|\lambda_{n}\right| \geqslant \varepsilon$, so that $\left\|u_{n}+\lambda_{n} x\right\|-1 \rightarrow \gamma \leqslant 0$. Clearly $\left|\lambda_{n}\right| \rightarrow \infty$ is impossible. Hence, after possible extraction of a subsequence, we can conclude that

$$
\left\|u_{n}+\lambda x\right\|-1 \rightarrow \gamma \leqslant 0
$$

for some $\lambda,|\lambda| \geqslant \varepsilon$. From the choice of the $f_{n}$ it follows that $u_{n}$ converges weakly $\left(\right.$ weak*) to 0 . By construction, $\left\|u_{n}\right\|=1$; however, by (7), $\left\|u_{n}-(-\lambda x)\right\|-1 \rightarrow \gamma \leqslant 0$. Since $\lambda x \neq 0$ this contradicts (3).

To show that the (weak*) approximate symmetry of $\perp$ implies ( $\left.3^{\prime}\right)$, we make only the obvious modifications in the first paragraph of this proof.

To show that the converse of the last assertion fails in general, we consider the space $X$ (due to Belluce, Kirk and Steiner [10]) which is $l_{2}$ renormed according to $|x|=\sup \left(1 / 2\|x\|_{2},\|x\|_{\infty}\right)$, where $\|x\|_{2}$ denotes the $l_{2}$ norm and $\|x\|_{\infty}$ denotes the $l_{\infty}$ norm. Choose $x=\left(\xi_{i}\right) \in X$ so that $\|x\|_{2} \leqslant 3 / 2,\|x\|_{\infty}$ $=1$, and $\xi_{i}<0$ all $i$. Choose $\varepsilon \leqslant 1 / 2$. Suppose $U=U(x, \varepsilon)$ satisfies (1) and (2). We can choose a finite dimensional subspace $V$ which is the complement of $U$. Hence we can express each $e_{n}=\left(\delta_{i n}\right)$ as $e_{n}=u_{n}+v_{n}$, with $u_{n} \in U$ and $v_{n} \in V$. Since $e_{n}$ converges weakly to 0 and $V$ is finite dimensional, we have that $\left\|v_{n}\right\| \rightarrow 0$ as $n \rightarrow \infty$. Thus $\left\|u_{n}\right\|_{2} \rightarrow 1,\left\|u_{n}\right\|_{\infty} \rightarrow 1$ and $\left|u_{n}\right|=\left\|u_{n}\right\|_{\infty}$ for sufficiently large $n$. Let $w_{n}=u_{n} /\left|u_{n}\right|+\varepsilon x$. It follows that $\left\|w_{n}\right\|_{2} \rightarrow \gamma \leqslant 1+$ $3 / 2 \varepsilon<2$. Recalling that $\xi_{i}<0$ for all $i$, it also follows that $\left|w_{n}\right|=\left\|w_{n}\right\|_{\infty}<1$, for $n$ sufficiently large. Thus $\left|u_{n} /\right| u_{n}|+\varepsilon x|<1$ for $n$ sufficiently large, which violates (2). Thus $\perp$ is not approximately symmetric.

Finally we show that $\left(3^{\prime}\right)$ holds. Suppose that $x_{n}$ converges weakly to 0 . We may assume that either $\lim \left\|x_{n}\right\|_{2}=2 r$ or that $\lim \left\|x_{n}\right\|_{\infty}=r$. In the former case, since $\perp$ is uniformly approximately symmetric in Hilbert space, we can apply (3) to conclude that $\lim \inf \left\|x_{n}-x\right\|_{2}>2 r$ for each $x \neq 0$ in $X$. Hence $\lim \inf \left|x_{n}-x\right|>r$, which implies $\left(3^{\prime}\right)$. In the latter case, it can be readily shown that if $x=\left(\xi_{i}\right)$ satisfies $\lim \inf \left\|x_{n}-x\right\|_{\infty} \leqslant r-\delta$, thın $\left|\xi_{i^{\prime}}\right| \geqslant \delta$ for an infinite sequence of integers $i^{\prime}$, whence $x \notin X$. This finishes the proof of Theorem 2.

Proof of Theorem 1, PART 2. Let $x \in C$ be arbitrarily chosen. Let $x_{n}=T^{n} x, n=1,2, \ldots$ Let $\left\{x_{n^{\prime}}\right\}$ be a weakly (weak*) convergent subsequence with limit $z$. Since, by hypothesis, $\left\|T x_{n^{\prime}}-x_{n^{\prime}}\right\| \rightarrow 0$, the proof of Theorem 1, part 1, is applicable, and it follows that $T z=z$. Next we note 
that $\left\|z-x_{n+1}\right\|=\left\|T z-T x_{n}\right\| \leqslant\left\|z-x_{n}\right\|$. Therefore $\lim \left\|x_{n}-z\right\|=r$ exists; and, by Theorem 2, $\lim \inf \left\|x_{n^{\prime}}-w\right\|>r$ for $w \neq z$. Now suppose that another subsequence $\left\{x_{n^{\prime \prime}}\right\}$ converges weakly (weak*) to $w \neq z$. Repeating the argument above we have $\lim \left\|x_{n}-w\right\|<\lim \inf \left\|x_{n^{\prime \prime}}-z\right\|=\lim \left\|x_{n^{\prime \prime}}-z\right\|$ $=r$, which is a contradiction. This finishes the proof of Theorem 1, part 2 .

\section{REFERENCES}

1. G. Birkhoff, Orthogonality in linear metric spaces, Duke Math. J. 1(1935), 169-172.

2. F. E. Browder, Nonexpansive nonlinear operators in a Banach space, Proc. Nat. Acad. Sci. U.S.A. 54(1965), 1041-1044. MR32 \#4574.

3. F. E. Browder and W. V. Petryshyn, The solution by iteration of nonlinear functional equations in Banach spaces, Bull. Amer. Math. Soc. 72(1966), 571-575. MR32 \#8155b.

4. D. Göhde, Uber Fixpunkte bei stetigen Selbstabbildungen mit kompakten Iterierten, Math. Nachr. 28(1964), 45-55. MR31 \#4020.

5. R. C. James, Orthogonality and linear functionals in normed linear spaces, Trans. Amer. Math. Soc. 61(1947), 265-292. MR9, 42.

6. A separable somewhat reflexive Banach space with nonseparable dual, Bull. Amer. Math. Soc. 80 (1974), 738-743.

7. W. A. Kirk, A fixed point theorem for mappings which do not increase distance, Amer. Math. Monthly 72(1965), 1004-1006. MR32 \#6436.

8. J. Lindenstrauss and C. Stegall, Examples of spaces which do not contain $l_{1}$ and whose duals are not separable (to appear).

9. Z. Opial, Weak convergence of the sequence of successive approximations for nonexpansive mappings, Bull. Amer. Math. Soc. 73(1967), 591-597. MR35 \#2183.

10. L. P. Belluce, W. A. Kirk and E. F. Steiner, Normal structure in Banach spaces, Pacific J. Math. 26(1968), 433-440. MR38 \#1501.

Institute for Fluid Dynamics and APPlied Mathematics, University of Maryland, College Park, Maryland 20742 